https://www.journal-imab-bg.org

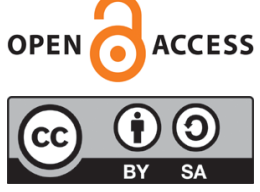

Original article

\title{
FACTORS WHICH INFLUENCE POSTOPERATIVE PAIN INTENSITY AFTER SURGICAL REMOVAL OF IMPACTED MANDIBULAR THIRD MOLARS
}

\author{
Tanya I. Sbirkova ${ }^{1}$, Deyan Z. Neychev ${ }^{1}$, Ralitsa D. Raycheva ${ }^{2}$, Dimitar T. \\ Atanasov ${ }^{1}$ \\ 1) Department of Oral Surgery, Faculty of Dental Medicine, Medical University \\ of Plovdiv, Bulgaria \\ 2) Department of Social Medicine and Public Health, Faculty of Public Health, \\ Medical University Plovdiv, Bulgaria.
}

\begin{abstract}
:
Introduction: Surgical removal of impacted mandibular third molars is one of the most common surgical procedures in oral surgery. Postoperative pain after such surgical procedures is acute and may last more than a week, thus impacting the patients' ability to perform activities of daily living.

Objective: The objective of this study is to elucidate the factors that have an effect on postoperative pain intensity.

Material and methods: A clinical study was conducted on 40 subjects at the age between 17 and 40 with bilaterally impacted mandibular third molars. The time interval between the two surgical procedures was 2 weeks. The patients were allocated by simple randomisation in the following groups depending on the medication taken: group A - placebo, group B - ibuprofen, group C - ibuprofen plus gabapentin. Visual analogue scale (VAS) was used to measure pain intensity. The following statistical methods were used to process the results: T-test, one-way analysis of variance (ANOVA), one-way ANOVA with repeated measures (RMA), post hoc tests and hierarchical multiple regression.

Results: The results of the study conducted show that the pain after both surgical procedures was most severe at postoperative hour 6; after the first operation the mean was $49.488 \pm 4.165 \mathrm{~mm}$, and after the second operation $39.566 \pm 4.935 \mathrm{~mm}$. A statistically significant difference (paired t-test, $\mathrm{p}=0.007, \mathrm{n}=38$ ) was found between the paired mean pain intensities measured at hour 6; the intensity was lower after the second procedure compared to the first one.

Conclusion: Our study showed that the factor with the greatest impact on the intensity of the postoperative pain was the difficulty of the surgical procedure.
\end{abstract}

Keywords: mandibular third molar, impacted teeth, postoperative pain

\section{INTRODUCTION:}

Each surgical procedure is associated with postoperative pain,which is acute in nature. According to literature data, about $80 \%$ of patients undergoing surgery experience moderate to severe postoperative pain.[1] Surgical removal of the mandibular third molar is one of the most common surgical procedures in oral surgery. [2] It is rarely life-threatening, but usually causes short-term changes in daily habits and in the quality of life of the patients in the postoperative period, [3] and the main factor for this is the presence of postoperative pain. [4]

NSAIDs are the most commonly used group of medications for postoperative pain management after surgical removal of mandibular third molars. [5] This group of medications has no effect on the normal pain threshold but reduces abnormal pain responses in inflammatory conditions. [6] In most patients, they have a satisfactory effect on postoperative pain. Ibuprofen is the most commonly used and investigated NSAID for relieving pain in dental practice as its efficacy is well documented, and it is safer compared to other similar medications. [7] In cases of surgical procedures with greater difficulty, which are accompanied by a more serious surgical trauma, NSAIDs are not effective enough in postoperative pain management, and it is necessary to prescribe medications from other medication groups. [8] Pain resulting from injury of peripheral nerve branches, which is the case in surgical procedures, is defined as neuropathic pain. [9] Anticonvulsants are medications of choice for neuropathic pain management. [10] Representatives of this group are gabapentin and pregabalin. [10]

\section{OBJECTIVE:}

The objectives of this study are to determine the predictors of the intensity of postoperative pain after surgical removal of impacted mandibular third molars and to examine the efficacy of the medications administered for managing this pain.

\section{PATIENTS AND METHODS:}

This study was conducted after obtaining approval from the Ethics Committee of the Medical University of Plovdiv, and all the patients who took part in it signed an informed consent form. 40 patients with indications for extraction of bilaterally impacted mandibular third molars were included in the study. This study was conducted be- 
tween September 2017 and May 2018 at the Department of Oral Surgery of the Faculty of Dental Medicine at the Medical University of Plovdiv.

Inclusion criteria: Clinically healthy patients between 17 and 40 years of age with no evidence of pain related to the mandibular third molar to be extracted at the time of the surgical procedure.

Exclusion criteria:Pregnancy and lactation, patients with a history of a head injury or seizures, neurological or psychiatric disorders, regular alcohol consumption and drug abuse, female patients 5 days before and 5 days after their menstrual period (in order to exclude the influence of hormonal factors on pain), a history of allergy to medications, and acute inflammation in the area of the tooth to be extracted.

Study design: This is a randomized, double-blind, placebo-controlled study. The patients were divided into three groups, depending on the medication administered: group A - placebo, group B - ibuprofen (400 mg) and group $\mathrm{C}-\mathrm{a}$ combination of ibuprofen $(400 \mathrm{mg}$ ) and gabapentin (300 mg). The patients were allocated to groups by simple randomisation, and each patient was randomly given a numbered vial (containing one of the above-mentioned medications). For greater credibility, the study was double-blind. Up to 30 minutes prior to the surgical procedure, each patient took one tablet of the medication in the numbered vial (preemptive analgesia). The medication was taken every 8 hours, up to postoperative hour 72. Each vial contained an envelope with three tablets, and the patients took one tablet at bedtime for three days, the first tablet is taken on the day of the surgical procedure. To this end, all subjects in the study received precise written and oral instructions on the use of the medications.

All patients included in the study underwent orthopantomography for diagnostic purposes, on the one hand, and for predicting the difficulty of the surgical procedure to be performed using Pederson index, on the other hand. [11] The difficulty of the surgical procedure was reported in both numerical and verbal forms, as follows: low (1-3 points), moderate (3-6 points) and great (7-10 points). The time interval between the two surgical procedures was 2 weeks. 2 of the patients withdrew in the second surgical procedure. The factors investigated as having an impact on postoperative pain were: duration and difficulty of the surgical procedure; age, sex and smoking status; presence of postoperative complications; and the type of medication administered, preemptive analgesia. To measure the intensity of postoperative pain, Visual Analogue Scale (VAS) was used, where the patient marked the perceived pain with a vertical check on a $100 \mathrm{~mm}$ ruler. Pain was reported at 3, 6, 24, 48, and 72 hours, respectively.

Surgical technique: The operations for surgical removal of mandibular third molars were performed using a standard technique, raising a triangular mucoperiosteal flap following anesthesia with $4 \%$ solution of articaine hydrochloride (Septanest). The bone covering the tooth was removed by rotary instruments and, if necessary, the tooth was separated into fragments. This was followed by dislocation and extraction of the tooth, smoothing of the bone edges, irrigation of the surgical wound with saline and placement of surgical suture, which was removed on postoperative day 7. If necessary, a prophylactic rubber drain was placed, and it was removed within 48 hours of surgery.

Statistical methods: Descriptive statistics was used to summarize quantitative data, to assess the main statistical parameters and to perform frequency analysis of qualitative variables expressed as percentages. All quantitative variables were tested for normal distribution with the nonparametric Kolmogorov-Smirnov test and with the ShapiroWilk test. Normally distributed data is represented as mean \pm standard error, while in the absence of normal distribution, the data is represented by positional averages - median (mdn) and interquartile range (IQR). In statistical hypothesis testing: we used paired t-test to compare the means of the dependent observations of the two groups; we used repeated measure analysis of variance (Repeated Measure ANOVA) to verify the equivalence of the means of more than two groups. Parameter estimation in regression models was based on hierarchical regression analysis so that to set a fixed order of input variables in order to control the effects of covariates or to test the effects of some predictors regardless of the impact of others. The critical level of significance used was $\alpha=0.05$. The respective zero hypothesis was rejected when the P-value was less than $\alpha$. Systematization, processing and analysis of data were performed with IBM SPSS Statistics (Statistical Package for the Social Sciences) v. 22.

\section{RESULTS:}

The mean age of the patients included in the study was $22 \pm 3$ years (19-38), 12 male patients $(30 \pm 7.24 \%)$ and 28 female patients $(70 \pm 7.24 \%) .29(72.5 \pm 7.06 \%)$ of the patients were non-smokers and $11(27.5 \pm 7.06 \%)$ were smokers, respectively. Non-uniform distribution by sex, age, and smoking status was observed.

The difficulty of the first surgical procedure was reported in two groups: moderate in $25 \pm 6.84 \%$ (10 subjects) and great in $75 \pm 6.84 \%$ (30 subjects), while in the second surgical procedure the distribution was as follows: moderate in $21.1 \pm 1.07 \%$ (8 subjects) and great in $78.8 \pm 1.07 \%$ (30 subjects). In both surgical procedures, we observed non-uniform distribution by this parameter, with mean difficulty in both procedures of $7 \pm 1$, which corresponds to surgical procedures with great difficulty.

The distribution of patients depending on the medication administered was as follows: in the first surgical procedure: group A - $20 \pm 6.32 \%$ ( 8 persons), group B - $35 \pm 7.54 \%$ (14 persons) and group $\mathrm{C}-45 \pm 7.86 \%$ (18 persons); in the

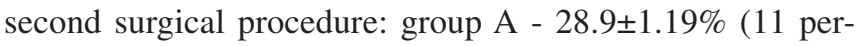
sons), group B $-39.5 \pm 1.28 \%$ (15 persons) and group C $31.6 \pm 1.22 \%$ (12 persons). The distribution of patients was non-uniform in both surgical procedures.

The mean duration of the surgical procedures was as follows: of the first surgical procedure - $25 \pm 22$ minutes, of the second surgical procedure- 35.26 \pm 13.262 minutes. Statistically significant longer duration of surgery in the second surgical procedures was found. In the first surgical procedure, non-uniform distribution was observed, unlike 
the second surgical procedure, where the distribution by duration of surgery was uniform.

Postoperative follow-up of patients was carried out, and all postoperative complications were described. After the first surgical procedure, complications occurred in $12.5 \pm 5.22 \%$ ( 5 cases), while after the second surgical procedure, complications were reported in $13.2 \pm 5.48 \%$ (5 cases), respectively.

For the first procedure, repeated-measures ANOVA with Greenhouse-Geisser correction showed a statistically significant difference in the means of pain intensity reported in the sample $(\mathrm{n}=40)$ between the different time points $(\mathrm{F}(2.063,10307.39)=14.079, \mathrm{p}=0.000)$. This demonstrates the high statistical significance of the results obtained. Post-hoc tests using the Bonferroni correction showed statistically significantly higher pain intensity at hour $6(49.488 \pm 4.165 \mathrm{~mm})$ vs. all other time points - hour $3,24,48,72(32.550 \pm 4.64 \mathrm{~mm}, \mathrm{p}=0.002,29.088 \pm 4.47 \mathrm{~mm}$, $\mathrm{p}=0.000,25.500 \pm 4.28 \mathrm{~mm}, \mathrm{p}=0.000,18.61 \pm 3.80 \mathrm{~mm}$, $\mathrm{p}=0.000$, respectively).

The pain reported at hour 72 after the first extraction was statistically significantly $(\mathrm{p}=0.018)$ lower than that reported at hour 24.

For the second procedure, the repeated measures ANOVA with Greenhouse-Geisser correction demonstrated a statistically significant difference in the means of pain intensity reported in the sample $(n=38)$ between the different time points $(\mathrm{F}(2.033,5240.53)=8.266, \mathrm{p}=0.001)$. Differences in mean pain intensity after the second surgical procedure were identical to those after the first surgical procedure, i. e. with a high level of statistical significance. Post-hoc tests using the Bonferroni correction showed statistically significantly higher pain intensity at hour 6 $(39.57 \pm 4.94 \mathrm{~mm}) \mathrm{vs}$. all other time points - hour $24,48,72$ $(23.28 \pm 4.37 \mathrm{~mm}, \mathrm{p}=0.011 ; 21.25 \pm 4.18 \mathrm{~mm}, \mathrm{p}=0.004$; $18.45 \pm 3.93 \mathrm{~mm}, \mathrm{p}=0.001$, respectively).

Fig. 1. Dynamics of the mean pain intensity measured by VAS after the first and the second extractions.

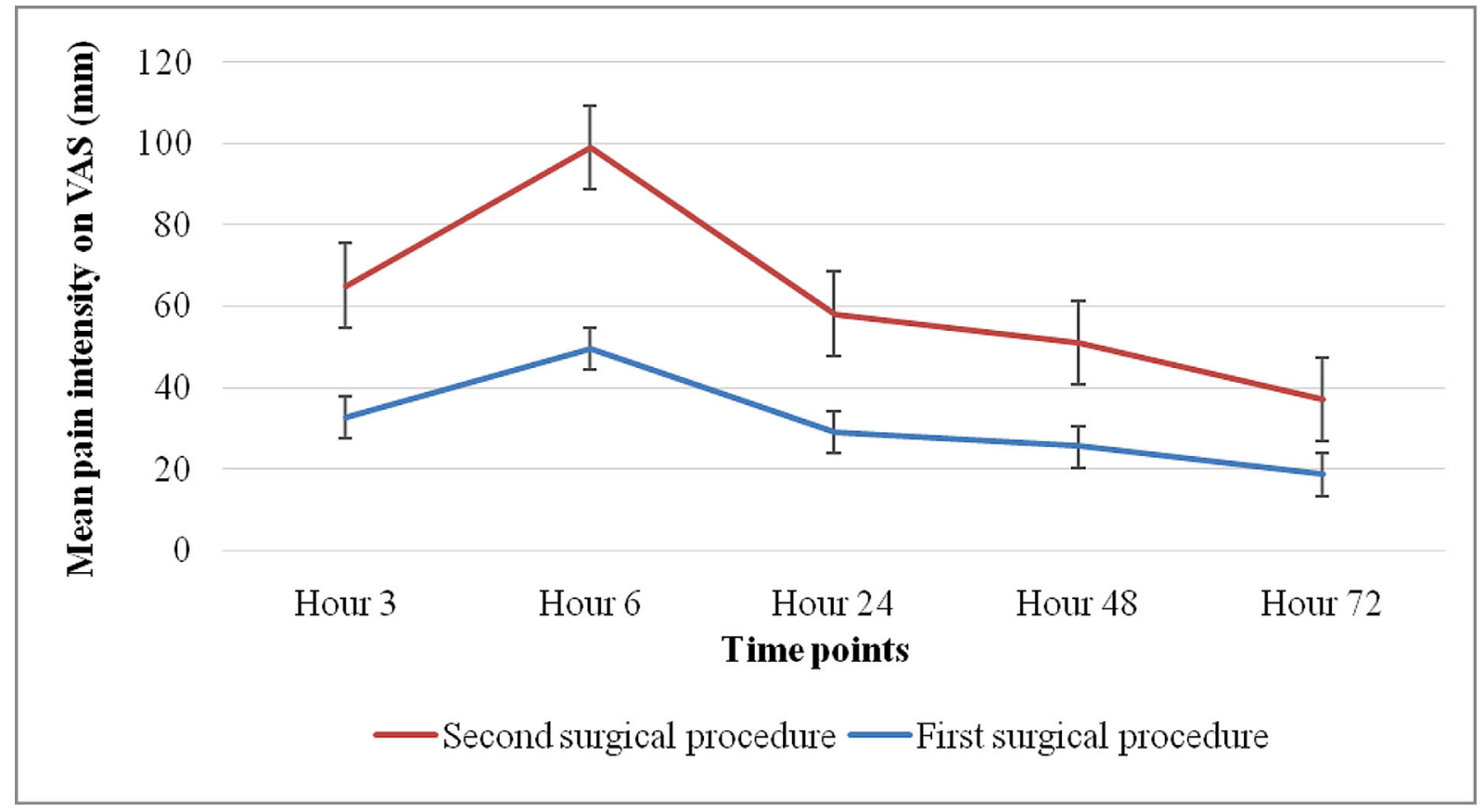

A statistically significant difference (paired t-test, $\mathrm{p}=0.007, \mathrm{n}=38$ ) was found between the paired mean pain intensities measured at hour 6; the intensity was statistically significantly lower after the second procedure $(39.57 \pm 4.94$ $\mathrm{mm})$ compared to the first one $(48.67 \pm 4.27 \mathrm{~mm})$.

Three-step hierarchical multiple regression was conducted with the pain reported at the respective hours in both procedures as a dependent variable: Step 1 - Here we included the predictors which we expected to predict the pain
- difficulty and duration of the procedure.

In the next steps, we included the explanatory predictor variables (stepwise method of inclusion) which we did not expect to necessarily affect the pain, as follows: Step 2 preemptive analgesia; Step 3 - complication; and Step 4 age, sex, and smoking status.

For the first extraction, this model gave results for hours 48 and 72, and for the second extraction - for hours 3, 24, 48 and 72 (Table 1.). 
Table 1. Summarized hierarchical regression analysis results for predictor variables for pain.

\begin{tabular}{|c|c|c|c|c|c|c|c|c|c|}
\hline Variable & b & SE & $\beta$ & $\mathbf{t}$ & p-value & $\mathbf{R}$ & $\mathbf{R}^{2}$ & adjusted $\mathbf{R}^{2}$ & $\Delta \mathbf{R}^{2}$ \\
\hline \multicolumn{10}{|c|}{ First surgical procedure } \\
\hline \multicolumn{10}{|l|}{ Pain at hour 48} \\
\hline \multicolumn{6}{|l|}{ Step 1} & 0.37 & 0.14 & 0.09 & \\
\hline Constant & -43.71 & 28.96 & & -1.51 & 0.140 & & & & \\
\hline Difficulty & 9.96 & 4.48 & 0.37 & 2.22 & 0.032 & & & & \\
\hline Duration & 0.02 & 0.28 & 0.01 & 0.08 & 0.936 & & & & \\
\hline \multicolumn{10}{|l|}{ Pain at hour 72} \\
\hline \multicolumn{6}{|l|}{ Step 1} & 0.49 & 0.24 & 0.20 & \\
\hline Constant & -56.32 & 24.24 & & -2.32 & 0.026 & & & & \\
\hline Difficulty & 9.72 & 3.75 & 0.40 & 2.59 & 0.014 & & & & \\
\hline Duration & 0.26 & 0.24 & 0.17 & 1.09 & 0.285 & & & & \\
\hline \multicolumn{10}{|c|}{ Second surgical procedure } \\
\hline \multicolumn{10}{|l|}{ Pain at hour 3} \\
\hline \multicolumn{6}{|l|}{ Step 1} & 0.22 & 0.05 & -0.01 & \\
\hline Constant & -7.17 & 28.90 & & -0.25 & 0.806 & & & & \\
\hline Difficulty & 4.55 & 4.80 & 0.20 & -.95 & 0.350 & & & & \\
\hline Duration & 0.09 & 0.45 & 0.04 & 0.19 & 0.849 & & & & \\
\hline \multicolumn{6}{|l|}{ Step 2} & 0.47 & 0.22 & 0.16 & 0.17 \\
\hline Constant & 33.42 & 30.28 & & 1.10 & 0.277 & & & & \\
\hline Difficulty & 3.81 & 4.41 & 0.16 & 0.87 & 0.393 & & & & \\
\hline Duration & -0.03 & 0.41 & -0.01 & -0.06 & 0.951 & & & & \\
\hline $\begin{array}{l}\text { Preemptive } \\
\text { analgesia }\end{array}$ & -15.43 & 5.57 & -0.43 & -2.77 & 0.009 & & & & \\
\hline \multicolumn{10}{|l|}{ Pain at hour 24} \\
\hline \multicolumn{6}{|l|}{ Step 1} & 0.45 & 0.20 & 0.16 & \\
\hline Constant & -47.28 & 24.91 & & -1.90 & 0.066 & & & & \\
\hline Difficulty & 9.28 & 4.14 & 0.43 & 2.25 & 0.031 & & & & \\
\hline Duration & 0.08 & 0.38 & 0.04 & 0.20 & 0.847 & & & & \\
\hline \multicolumn{10}{|l|}{ Pain at hour 48} \\
\hline \multicolumn{6}{|l|}{ Step 1} & 0.53 & 0.28 & 0.24 & \\
\hline Constant & -58.73 & 22.64 & & -2.59 & 0.014 & & & & \\
\hline Difficulty & 10.68 & 3.76 & 0.51 & 2.84 & 0.007 & & & & \\
\hline Duration & 0.05 & 0.35 & 0.03 & 0.15 & 0.880 & & & & \\
\hline \multicolumn{6}{|l|}{ Step 2} & 0.61 & 0.38 & 0.32 & 0.10 \\
\hline Constant & -47.28 & 21.94 & & -2.16 & 0.038 & & & & \\
\hline Difficulty & 8.44 & 3.68 & 0.40 & 2.30 & 0.028 & & & & \\
\hline Duration & 0.10 & 0.33 & 0.05 & 0.30 & 0.763 & & & & \\
\hline Complication & 24.49 & 10.64 & 0.33 & 2.30 & 0.028 & & & & \\
\hline \multicolumn{10}{|l|}{ Pain at hour 72} \\
\hline \multicolumn{6}{|l|}{ Step 1} & 0.59 & 0.35 & 0.31 & \\
\hline Constant & -65.22 & 20.24 & & -3.22 & 0.003 & & & & \\
\hline Difficulty & 11.09 & 3.36 & 0.57 & 3.30 & 0.002 & & & & \\
\hline
\end{tabular}




\begin{tabular}{|l|r|r|r|r|r|r|r|r|l|}
\hline Duration & 0.07 & 0.31 & 0.04 & 0.23 & 0.818 & & & & \\
\hline Step 2 & -55.43 & 19.75 & & -2.81 & 0.008 & & & & 0.80 \\
\hline Constant & 9.18 & 3.31 & 0.47 & 2.77 & 0.009 & & & & \\
\hline Difficulty & 0.11 & 0.30 & 0.06 & 0.38 & 0.706 & & & & \\
\hline Duration & 20.92 & 9.57 & 0.30 & 2.19 & 0.036 & & & & \\
\hline Complication &
\end{tabular}

The use of hierarchical multiple regression shows that in the first procedure the difficulty of the surgery contributes significantly to the regression model at hour 48 $\mathrm{F}(2.37)=3.05, \mathrm{p}=0.041$, and hour $72 \mathrm{~F}(2.37)=5.82, \mathrm{p}=0.006$, determining $14 \%$ and $24 \%$ of the pain intensity changes at these time points, respectively. The regression coefficient for hour $48-b_{0}=-43.71$ (constant) is not statistically significant $(\mathrm{t}=1.51, \mathrm{p}=0.140)$, whereas the regression coefficient in front of the factor variable - difficulty $b_{1}=9.96$ is $(\mathrm{t}=2.22, \mathrm{p}=0.032)$ and is significant. For hour 72 , the regression coefficient $b_{0}=-56.32$ is already statistically significant $(\mathrm{t}=2.32, \mathrm{p}=0.026)$, as well as $\mathrm{b}_{1}=9.72(\mathrm{t}=2.59$, $\mathrm{p}=0.014)$.

At both time points, stepwise regression excludes the variables of Steps 2, 3 and 4 from the regression equation as non-essential, i.e. regression coefficients in front of these factors are not statistically significant; therefore, they do not have a significant influence on pain intensity.

For the second procedure, the hierarchical multiple regression demonstrates the adequacy of the model at: (I) hour 3 after addition of predictor variable - preemptive analgesia $F(3.34)=3.28, p=0.033$, which is statistically significant; The inclusion of this variable explains an additional $16 \%$ of the variation in pain intensity, with only its regression coefficient $b_{3}=-15.43$ being statistically significant $(\mathrm{t}=2.77, \mathrm{p}=0.009)$. One-way analysis of variance (ANOVA) with Bonferroni correction showed statistically significantly lower levels of pain at hour 3 in Group B $(n=15$; $23.00 \pm 6.30 \mathrm{~mm}$ ) treated with ibuprofen, and in group $\mathrm{C}$ $(\mathrm{n}=12 ; 17.41 \pm 5.63 \mathrm{~mm})$ treated with combination of ibuprofen with gabapentin compared to group A $(n=11$; $50.27 \pm 9.84 \mathrm{~mm}$ ) who received placebo, $\mathrm{p}=0.034$ and $\mathrm{p}=0.013$, respectively;

(II) hour $24 \mathrm{~F}(2.35)=4.41, \mathrm{p}=0.020$ only in Step 1 and excluding the variables in Steps 2, 3 and 4, with difficulty explaining $20 \%$ of the variation in pain. The regression coefficient is statistically significant $(\mathrm{t}=2.25, \mathrm{p}=0.031)$ only in front of the factor variable - difficulty $b_{1}=9.28$. One-way analysis of variance (ANOVA) with Bonferroni correction demonstrates a statistically significant difference between the mean pain scores $(\mathrm{p}=0.020)$ at hour 24 with Pederson difficulty index $6(\mathrm{n}=5 ; 4.00 \pm 23.10 \mathrm{~mm})$ versus Pederson difficulty index $9(\mathrm{n}=4 ; 58.25 \pm 12.48 \mathrm{~mm})$;

(III) hours 48 and 72, at which the difficulty in Step 1 contributes significantly to the adequacy of the regression models $-\mathrm{F}(2.35)=6.77, \mathrm{p}=0.003$ and $\mathrm{F}(2.35)=9.32$, $\mathrm{p}=0.001$, respectively, explaining $28 \%$ and $35 \%$ of the variation in pain. For both time points, in Step 2, introducing the variable complication from Step 3 represents an additional $10 \%$ and $8 \%$ of variation; this change in $\mathrm{R}^{2}$ is sig- nificant $-\mathrm{F}(3.34)=6.83, \mathrm{p}=0.001$ at hour 48 and $\mathrm{F}(3.34)=$ $8.48, \mathrm{p}=0.000$ at hour 72 . In patients with complications, pain intensities at these time points are statistically significantly higher $(\mathrm{n}=5 ; 55.40 \pm 16.04 \mathrm{~mm})$ at hour 48 and $(\mathrm{n}=5 ; 45.20 \pm 16.14 \mathrm{~mm})$ at hour 72 compared to patients without complications $(\mathrm{n}=33,16.83 \pm 3.70 \mathrm{~mm})-\mathrm{t}=3.00$, $\mathrm{p}=0.005$ at hour 48 and $(\mathrm{n}=33,14.39 \pm 3.42 \mathrm{~mm})-\mathrm{t}=2.91$, $\mathrm{p}=0.006$ at hour 72 , respectively.

At hour 48 , the values of the standardized regression coefficient $\beta$ for the two prediction variables - difficulty $(\beta=0.40)$ and complication $(\beta=0.33)$ are close to each other. Therefore, we can conclude that they have a similar effect on the pain at this time point. On the other hand, at hour 72 , the standardized regression coefficient $\beta$ for difficulty $(\beta=0.47)$ is higher than that for complications $(\beta=0.30)$, therefore the difficulty of the procedure has a greater impact on the pain intensity compared to the occurrence of a complication.

\section{DISCUSSION:}

Postoperative pain after surgical removal of impacted mandibular third molars accompanies each surgical procedure. It is usually acute, with short duration (from 2 hours to 3 days) and reaches its maximum intensity immediately after the procedure. [12]

In our study, we found that the highest intensity of postoperative pain in both surgical procedures was at postoperative hour 6 , and it was statistically different from the pain intensity measured at the other hours reported (hour $3,24,48$ and 72, respectively). Thus, the results obtained by us are different from the data of other authors. Chugh A, et al. [13] reported that the maximum pain intensity after surgical removal of a mandibular third molar was at 4872 postoperative hours, while Bocanegra $\mathrm{M}$, et al. [12] found in a study that the maximum intensity of postoperative pain was in the first 4 postoperative hours.

The pain after the second surgical procedure at hour 6 was statistically significantly milder than the pain at the same hour after the first surgical procedure, although the mean difficulty in both groups of surgical procedures was reported to be the same - great difficulty. The second surgical procedures were longer, and more complications occurred during their implementation compared to the first surgical procedures. Benediktsdóttir IS, et al. [14] found in a study that $15.2 \%$ of the patients experienced more severe pain than they expected during the first postoperative week. The pain after the second procedure was milder at hour 6, which can be explained by the fact that the patients having undergone the first operation are prepared what to expect in the postoperative period and deal more easily 
with postoperative pain.

Noxious stimuli that are strong enough to induce tissue damage can cause hypersensitivity, hyperalgesia, allodynia and unusual paresthesia, due to which pain can be induced even by non-invasive stimuli. This can be explained by the combination of peripheral sensitization associated with the lower nociceptive threshold and central sensitization associated with higher excitability of the central nervous system. Uncontrolled postoperative pain is also thought to be the cause of such type of sensory disorders. After central sensitization occurs due to surgical damage of tissue, postoperative hyperesthesia is prolonged, and it takes additional time to improve. Preemptive analgesia minimizes postoperative pain by suppressing central sensitization, which prevents the development of postoperative hyperesthesia. [15] In our study with preemptive analgesia, patients received placebo or ibuprofen. The results obtained clearly demonstrate that after the second surgical procedure, the pain intensity measured at postoperative hour 3 was lower in the groups on ibuprofen compared to the placebo group. These results demonstrate the efficacy of preemptive analgesia following surgical removal of impacted mandibular third molars. Similar results were reported by authors working on the problem of the efficacy of preemptive analgesia. [16, 17, 18]

Ibuprofen is a medication from the group of NSAIDs. In 2015, in a study, Hodkinson DJ, et al. [6] found that this group of medications have an effect on the inflammatory component of pain only, but not on the neuropathic component of pain. This necessitates the inclusion of another group of medications for postoperative pain management. In our study, the patients in group $\mathrm{C}$, besides Ibuprofen, also received medication from the group of anticonvulsants (gabapentin). The statistical analysis of the results found no difference in postoperative pain response in the different patient groups, which, in our opinion, can be explained by the fact that pain intensity was not measured at hour 12 when the effect of gabapentin administration would be evident. In our opinion, the absence of statistically reliable data on the effect of the medication administered may be due to the influence of the cognitive component of the pain.

Based on the results from the statistical analysis, we can conclude that the main predictor of postoperative pain after surgical removal of impacted mandibular third molars using chisel and mallet technique is the difficulty of the surgical procedure. In both surgical procedures, the pain and difficulty at hours 48 and 72 were directly proportional. This fact can also be confirmed by other authors who worked on the problem and who found that the more curved the roots of the third molars are, the higher the postoperative pain intensity reported by the patients is. [14] Morecurved roots are associated with greater trauma due to the surgical procedure.

The factors duration, sex, age and smoking status do not influence the postoperative pain intensity according to our statistical analyses. In contrast to the results of our study, some authors claim that the main factor influencing postoperative complications and pain is the duration of the surgical procedure. [19] Benediktsdóttir IS, et al. [14] claimed that the factors sex, age and smoking status increase the risk of complications and postoperative pain. In a study, they found sex differences in the sensation of pain and pointed out that female patients reported postoperative pain after extraction of mandibular third molars 3 times more than male patients. [14] In the same study, the authors found that age had a role for postoperative pain intensity; the older the patients, the longer the surgical procedures were, and the more intensive the postoperative pain reported was. In our study, it was not possible to analyze the age as a factor due to the similar age of the participants.

Our results show that after the second surgical procedure, at hours 48 and 72, the factor complication had an important influence on the postoperative pain intensity. For the first surgical procedure, this fact cannot be confirmed, so the question of the influence of postoperative complications on the intensity of postoperative pain remains controversial.

\section{CONCLUSION:}

The findings of our study show that the strongest predictor of postoperative pain after surgical removal of impacted mandibular third molars is the difficulty. The preemptive analgesia used by us reduced the intensity of postoperative pain, which could justify its more frequent administration, especially in cases of surgical procedures with great difficulty.

\section{REFERENCES:}

1. Apfelbaum JL, Chen C, Mehta SS, Gan TJ. Postoperative pain experience: Results from a national survey suggest postoperative pain continues to be undermanaged. Anesth Analg. 2003 Aug; 97(2):534-40 [PubMed][Crossref]

2. Canellas JVDS, Ritto FG, Medeiros PJD. Evaluation of postoperative complications after mandibular third molar surgery with the use of platelet-rich fibrin: a systematic review and meta-analysis. Int J Oral
Maxillofac Surg. 2017 Sep;46(9): 1138-1146. [PubMed] [Crossref]

3. Matijevic $M$, Uzarevic $Z$, Ivanisevic Z, Gvozdic V, Leovic D, Popic B, et al. Determining the Quality of Life after Removing of Impacted Lower Wisdom Tooth using the Principal Component Analysis Method. Coll Antropol. 2014 Jun;38(2):691-9. [PubMed]

4. Hanna K, Sambrook P, Armfield JM, Brennan DS. Exploring and mod- elling impacts of third molar experience on quality of life: a real-time qualitative study using Twitter. Int Dent J. 2017 Oct;67(5):272-280. [PubMed] [Crossref]

5. Peterson L, Ellis III E, Hupp J, Tucker M.Contemporary oral and maxillofacial surgery. Saint Louis: Elsevier Mosby. 2013;126-45.

6. Hodkinson DJ, Khawaja N, O'Daly O, Thacker MA, Zelaya FO, Wooldridge CL, et al. Cerebral analge- 
sic response to nonsteroidal anti-inflamatory drug ibuprofen. Pain. $2015 \mathrm{Jul}$; 156(7):1301-10 [PubMed] [Crossref]

7. Smith EA, Marshall JG, Selph SS, Barker DR, Sedgley CM. Nonsteroidal Anti-inflammatory Drugs for Managing Postoperative Endodontic Pain in Patients Who Present with Preoperative Pain: A Systematic Review and Metaanalysis. J Endod. 2017 Jan;43(1):7-15. [PubMed] [Crossref]

8. Zupelari-Goncalves P, Weckwerth GM, Calvo AM, Simoneti LF, Dionisio TJ, Brozoski DT, et al. Efficacy of oral diclofenac with or without codeine for pain control after invasive bilateral third molar extractions. Int J Oral Maxillofac Surg. 2017 May;46(5):621-627. [PubMed] [Crossref]

9. Alles SRA, Bandet MV, Eppler K, Noh MC, Winship IR, Baker G, et al. Acute anti-allodynic action of gabapentin in dorsal horn and primary somatosensory cortex: Correlation of behavioural and physiological data. Neuropharmacology. 2017 Feb;113(Pt A):576-590 [PubMed] [Crossref]

10. Finnerup NB, Attal N, Haroutounian S, McNicol E, Baron R, Dworkin RH, et al. Pharmacotherapy for neuropathic pain in adults: a systematic review and meta-analysis.
Lancet Neurol. 2015 Feb;14(2):162-73

[PubMed] [Crossref]

11. Pederson GW. Surgical removal of tooth. In: Pederson GW, editor. Oral surgery. Philadelphia: WB Saunders; 1988

12. Bocanegra M, Seijas A, Yibirin MG. Effectiveness and tolerability of once-daily Nimesulid versus Ibuprofen in pain management after surgical extraction of an impacted third molar : a 24- hour, double-blind, randomized, double-dummy, parallel- group study. Curr Ther Res Clin Exp. 2005 May; 66(3):172-80 [PubMed] [Crossref]

13. Chugh A, Singh S, Mittal Y, Chugh V. Submucosal injection of dexamethasone and methylprednisolone for the control of postoperative sequelae after third molar surgery: randomized controlled trial. Int J Oral Maxillofac Surg. 2018 Feb;47(2):228233. [PubMed] [Crossref]

14. Benediktsdóttir IS, Wenzel A, Petersen JK, Hintze H. Mandibular third molar removal: Risk indicators for extended operation time, postoperative pain, and complications. Oral Surg Oral Med Oral Pathol Oral Radiol Endod. 2004 Apr;97(4):438-46. [PubMed] [Crossref]

15. Yamaguchi A, Sano K. Effec- tiveness of preemptive analgesia on postoperative pain following third molar surgery: Review of literatures. Japan Dent Scien Rev. 2013 Nov; 49(4):131-138 [Crossref]

16. Zor ZF, Isik B, Cetiner S. Efficacy of preemptive lornoxicam on postoperative analgesia after surgical removal of mandibular third molars. Oral Surg Oral Med Oral Pathol Oral Radiol. 2014 Jan;117(1):27-31 [PubMed] [Crossref]

17. Gazal G, Al-Samadani KH. Comparison of paracetamol, ibuprofen, and diclofenac potassium for pain relief following dental extractions and deep cavity preparations. Saudi Med J. 2017 Mar;38(3):284-291 [PubMed] [Crossref]

18. Silva de Oliveira JC, Grossi de Oliveira GA, Bassi APF. Comparative Assessment of the Effect of Ibuprofen and Etodolac on Edema, Trismus, and Pain in Lower Third Molar Surgery: A Randomized Clinical Trial. J Oral Maxillofac Surg. 2016 Aug;74(8): 1524-30. [PubMed] [Crossref]

19. Conrad SM, Blakey GH. Patients' perception of recovery after third molar surgery. J Oral MaxillofacSurg. 1999 Nov;57(11): 1288-94. [PubMed] [Crossref].

Please cite this article as: Sbirkova TI, Neychev DZ, Raycheva RD, Atanasov DT. Factors which Influence Postoperative Pain Intensity after Surgical Removal of Impacted Mandibular Third Molars. J of IMAB. 2019 Oct-Dec;25(4):27932799. DOI: https://doi.org/10.5272/jimab.2019254.2793

Received: 21/03/2019; Published online: 25/11/2019

\author{
Address for correspondence: \\ Tanya Sbirkova \\ Department of Oral Surgery, Faculty of Dental Medicine, Medical University \\ of Plovdiv, \\ 3, Hristo Botev blvd., Plovdiv 4000, Bulgaria. \\ E-mail: tanya_sbirkova@abv.bg
}

